2016-08-12

\title{
Gold Nanostars for Efficient In Vitro and In Vivo Real-time SERS Detection and Drug Delivery via Plasmonic-tunable Raman/FTIR Imaging
}

\author{
Furong Tian \\ Technological University Dublin, furong.tian@tudublin.ie \\ Joao Conde \\ Massachusetts Institute of Technology, jdconde@mit.edu \\ Chenchen Bao \\ Shanghai Jiaotong University
}

See next page for additional authors

Follow this and additional works at: https://arrow.tudublin.ie/schfsehart

Part of the Nanomedicine Commons

\section{Recommended Citation}

Tian, F., Conde J., Bao, C. Chen, Y., Curtin, J. \& Cui, D. (2016). Gold nanostars for efficient in vitro and in vivo real-time SERS detection and drug delivery via plasmonic-tunable Raman/FTIR imaging. Biomaterials, vo.106, pp.87-97.

This Article is brought to you for free and open access by the School of Food Science and Environmental Health at ARROW@TU Dublin. It has been accepted for inclusion in Articles by an authorized administrator of ARROW@TU

Dublin. For more information, please contact

arrow.admin@tudublin.ie, aisling.coyne@tudublin.ie, gerard.connolly@tudublin.ie.

Funder: Science Foundation Ireland and FP7

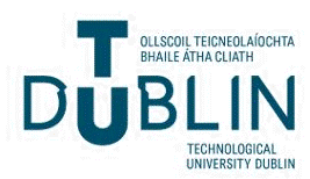




\section{Authors}

Furong Tian, Joao Conde, Chenchen Bao, Yunsheng Chen, James Curtin, and Daxiang Cui 


\section{Gold Nanostars for efficient In Vitro and In Vivo Real- Time SERS Detection and Drug Delivery via Plasmonic- Tunable Raman/FTIR Imaging}

Furong Tian ${ }^{1, *}$, João Conde ${ }^{2,3, *}$, Chenchen $\mathrm{Bao}^{4,5}$, Yunsheng Chen ${ }^{4}, \mathrm{James}_{\text {Curtin }}{ }^{1}$ and Daxiang Cui ${ }^{4}$

${ }^{1}$ School of Food Science and Environmental Health, College of Sciences and Health, Dublin Institute of Technology, Dublin, Ireland.

${ }^{2}$ Massachusetts Institute of Technology, Institute for Medical Engineering and Science, Harvard-MIT Division for Health Sciences and Technology, Cambridge, Massachusetts, USA.

${ }^{3}$ School of Engineering and Materials Science, Queen Mary University of London, London, UK.

${ }^{4}$ Department of Bio-Nano Science and Engineering, Dept. of Instrument Science and Engineering, School of Electronic Information and Electrical Engineering, Shanghai Jiao Tong University, P.R.China.

${ }^{5}$ United Well Technology Limited, Shanghai, China

* Corresponding authors, Furong Tian furong.tian@dit.ie and João Conde jdconde@ mit.edu

Keywords: gold nanostars; theranostics; drug delivery; in vivo SERS; Raman/FTIR imaging.

\section{ABSTRACT}

The application of plasmonicenhanced Raman imaging of cancer cells and drug delivery is gaining momentum. Here, we propose a new theranostic strategy based on an efficient plasmonic-tunable

Raman/Fourier transform infrared (FTIR) spectroscopy imaging, to simultaneously evaluate the anticancer drug scattering cellular imaging and the Raman scattering molecular
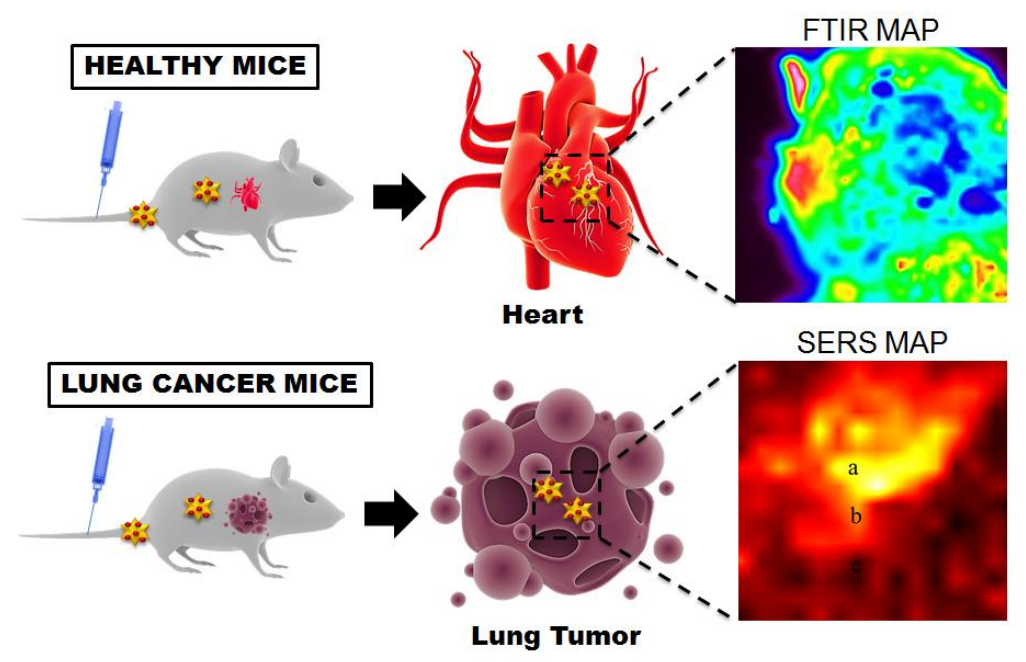
vibration signals in living cells. This technique allows to monitoring the drug release throughout the cell cycle and in vivo biodistribution and biocompatibility with low dose drug therapy $(200 \mu \mathrm{g} / \mathrm{mL})$ and low toxicity effect. This system can directly track in real-time the delivery and release of an anticancer drug (mitoxantrone, MTX) from gold nanostars in single 
living cells and in mice (healthy and lung cancer mice models), revealing a strong accumulation in the heart of healthy mice 5 minutes after administration and infiltration in the tumor site of lung cancer mice 5 hours after systemic injection. This in vivo SERS detection method holds a great promise for application in image-guided cancer chemotherapy or as a nonspecific anti-inflammatory therapy for patients with cardiovascular diseases or chronic heart failure.

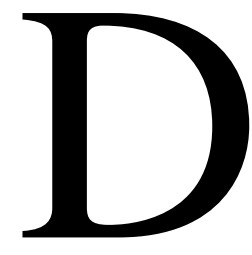

rug delivery systems using nanomaterials have been widely used to improve drug efficiency, reducing the dosage and minimizing side effects. ${ }^{1,2}$ However, not every drug can be successfully conjugated, tracked and delivered into target organism. For example, pharmacokinetic analyses of nanoparticles conjugated with an anticancer drug such as doxorubicin do not give information about the position, area, and size of drugs within tissue due to cell toxicity and its fluorescence wavelength overlaps with the tissue. ${ }^{12}$ Owing to their biocompatibility, feasible surface modification and tiny size, gold nanoparticles (GNPs) have been used for drug delivery, tumor targeting, gene therapy and photothermal therapy. ${ }^{5-11}$ However, Raman signals are inherently weak for in vivo imaging and drug location tracking. GNPs can provide broad wavelength for Raman scattering. Since it is a challenge to avoid auto-fluorescence and minimize light absorption from tissue, one way to amplify weak Raman signals is to employ Surface-enhanced Raman spectroscopy (SERS) using sharp ending metal surfaces, such as nanostars. ${ }^{5}$ As a result of utilizing different shape plasmonic-enhanced Raman signal from anticancer drugs, it has been possible to obtain a real-time cellular imaging of cancer cells.

Recently, our group has developed a protocol to synthesize gold nanostars to enhance the Raman signal using SERS. ${ }^{13}$ We found that it is necessary to optimize several conditions and physical properties of nanostars to visualize anticancer drugs inside cancer cells and organs. By optimizing these conditions gold nanostars can tune the wavelength of gold nanospheres from $560 \mathrm{~nm}$ to the range between ultraviolet and infrared regions. ${ }^{14,15}$ For example, Hirsch $e t$ 
al. have employed nanoshells to tune a wavelength of gold nanoparticles at $720 \mathrm{~nm}$ to achieve photothermal ablation of tumor cells by irradiation using near-infrared laser light in vivo. ${ }^{16}$

Gold nanostars, star-shaped gold nanoparticles, have exceptional optical properties. ${ }^{17-20}$ They display two surface plasmon bands consistent to the transverse and longitudinal surface plasmon bands in the visible $(520 \mathrm{~nm})$ and the near-infrared regions, respectively ${ }^{15}$ Thus, gold nanostars are special materials with an intense surface plasmon band that affords absorption, fluorescence $^{21-23}$ and light scattering ${ }^{24,25}$ in the near-infrared region, inducing two-photon luminescence phenomena. ${ }^{26}$

Hexadecyltrimethylammonium bromide $(\mathrm{CTAB})$ is a common material for gold nanostar synthesis. CTAB provides a cationic detergent to stabilizing agent during the preparation of gold nanostars. ${ }^{27}$ The disadvantage of using CTAB method, the toxicity is not only come from CTAB, but also from silver ions. ${ }^{28}$ Reducing concentration of CTAB during synthesis will cause the aggregation of nanostar. HEPES buffers (3-[4-(2-hydroxyethyl)piperazine-1yl]propane-1-sulphonic acid) have been used commonly used in cell culture and work as a functional reducing agent to directly change the shape of spherical gold nanoparticles to star shape. ${ }^{29}$ Using this method we were able to produce three-dimensional branched gold nanostars and drug-nanostars.

In the present work, we proposed a new theranostics strategy using Raman/FTIR imaging spectroscopy to track in real-time the delivery and release of an anticancer drug from nanostars in living cells and their biodistribution in vivo. Mitoxantrone (MTX) ${ }^{12}$ was conjugated to the surface of gold nanostars via a thiolated and carboxylated poly(ethylene glycol) (PEG) spacer that was attached by one-step ligand exchange and that provided the anchoring moieties for the covalent binding of amine-containing molecules like the MTX through carbodiimide chemistry (EDC/NHS coupling). MTX is a hydrophobic drug, slightly soluble in water, but with a high solubility in lipids as previously demonstrated. ${ }^{30}$ With a lipid/water partitioning coefficient of around 230,000 , it is rational to undertake that MTX 


\section{BIOMATERIALS}

crosses the plasma membrane at a high rate. ${ }^{31}$ The MTX is an anticancer drug with significant clinical activity. Furthermore, MTX has antiviral, antibacterial, antiprotozoal, immunomodulating, and antineoplastic properties and is mutagenic in some animal systems. ${ }^{32}$ Moreover, MTX has a red fluorescence emission as well as a strong Raman scattering signal with gold nanoparticles. The Raman signals of MTX molecules were evaluated by the plasmonic field of GNPs, depending on the distance between the drug molecules and the nanoparticle's surface. This technique allowed the simultaneous detection of the anticancer drug scattering cellular imaging and the biomolecules in living cells and in two mice models heart tissue imaging and drug accumulation in healthy mice and tumor imaging in a lung cancer mice model (see Figure 1). Therefore, gold nanostars can be used as a theranostic platform for efficient intracellular and in vivo SERS detection combined with real-time drug delivery using Raman/FTIR imaging, especially for image-guided cancer chemotherapy or other diseases. 

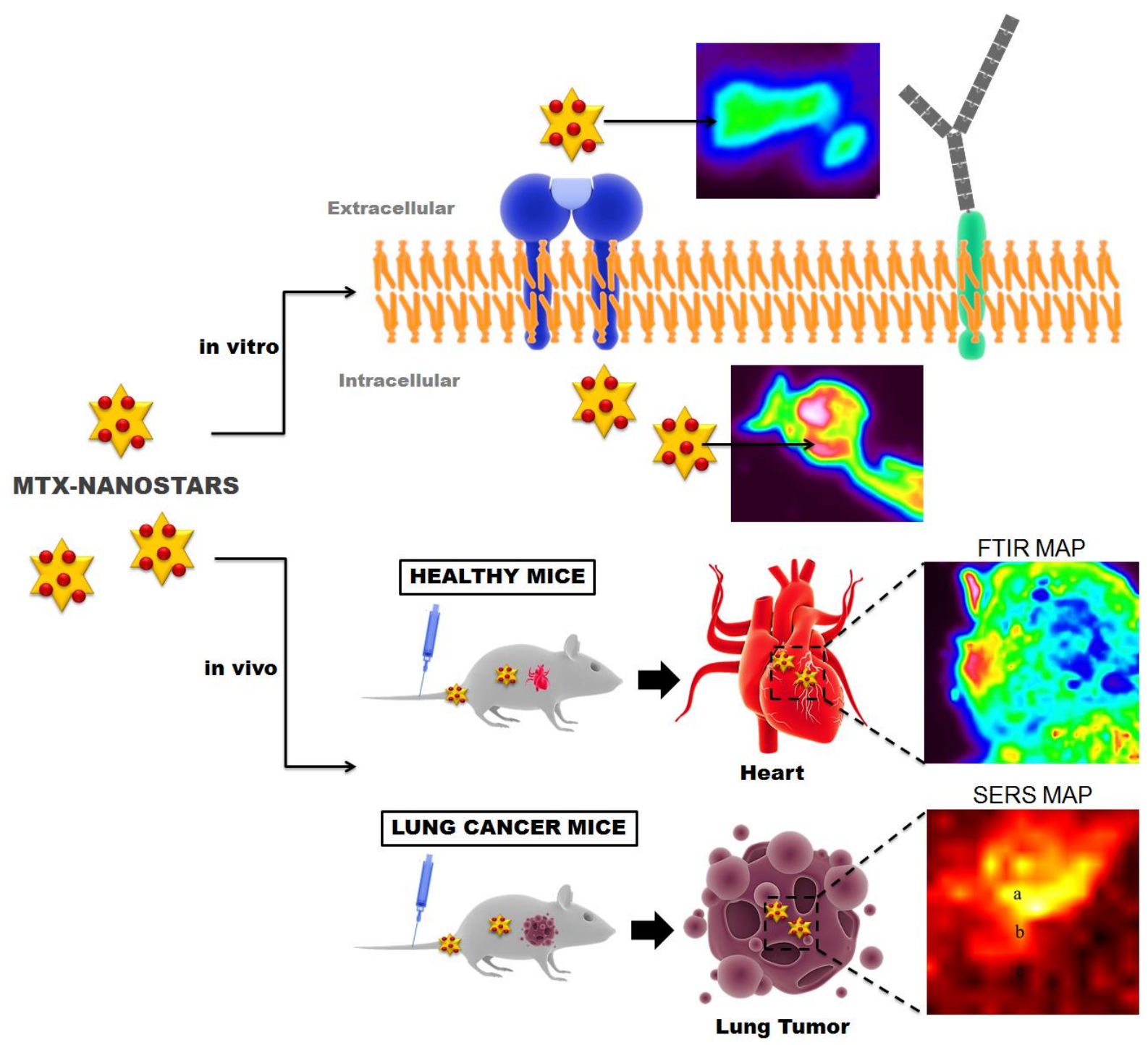

Figure 1. Gold Nanostars for theranostics: intracellular and in vivo SERS detection combined with real-time drug delivery using plasmonic-tunable Raman/FTIR imaging. This system can directly track in real-time the delivery and release of an anticancer drug (mitoxantrone - MTX) from gold nanostars in single living cells and in mice (healthy and lung cancer mice models). This in vivo SERS detection method holds a great promise for application in image-guided cancer chemotherapy or as a nonspecific anti-inflammatory therapy for patients with cardiovascular diseases or chronic heart failure.

\section{RESULTS AND DISCUSSION}

The application of plasmonic-enhanced Raman signal from an anticancer drug conjugated to gold nanostars in real-time imaging of cancer cells and drug delivery is gaining momentum. ${ }^{12}$ In order to enhance pharmacokinetic analyses of nanoparticles conjugated with an anticancer drug, gold nanostars were employed in the current study. 
The gold nanostars were synthesized via seed mediated route using HEPES buffer and conjugated with a thiolated and carboxylated poly(ethylene glycol) (PEG) spacer that provided the functional groups for the covalent binding of amine-containing MTX (2 amine groups) through carbodiimide chemistry (EDC/NHS coupling) (Figure 2A). See supporting information for detailed description about the functionalization and quantification of PEG and MTX on the surface of the nanostars (Supporting Information Figures S1-S2 and Table S1). Briefly, The final PEG:Nanostar molar ratio was around 600:1 and the MTX:Nanostar was 100:1 (see Table S1, Supporting Information).

Electron microscopy was employed to confirm the change in the dynamic light scattering profile of the nanospheres. Figure 2B shows electron microscopy images of $10 \mathrm{~nm}$ gold nanospheres used as seeds for the synthesis of gold nanostars. The synthesized nanostars presented a central core and six vertices in a three-dimensional arrangement, with an average diameter of approximately $35 \mathrm{~nm}$ (Figure 2C). 
$\mathbf{A}$
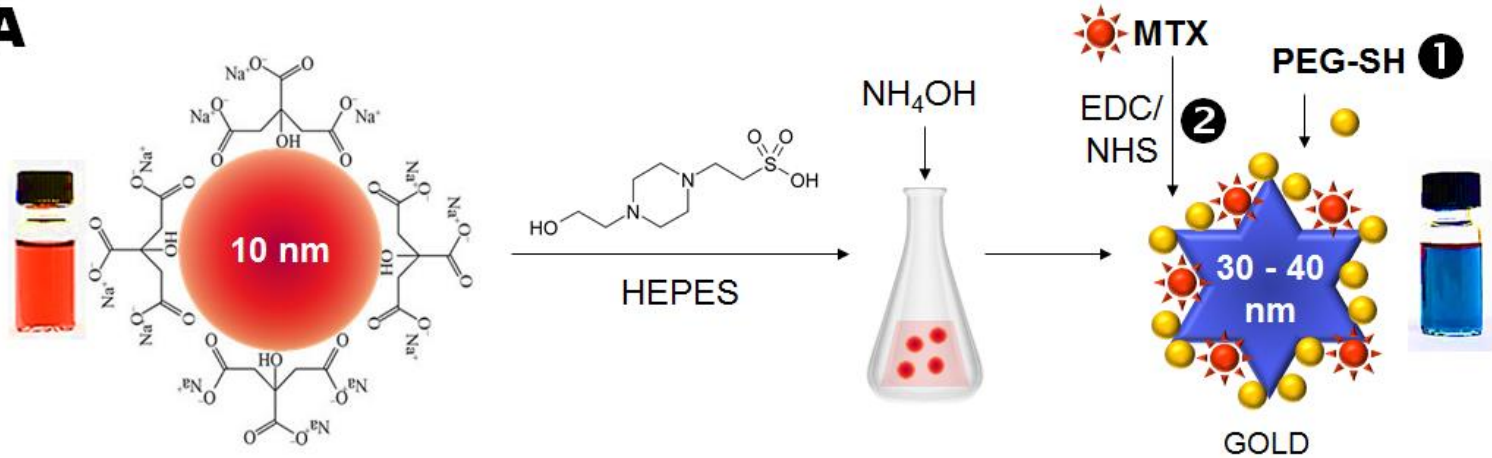

GOLD

CITRATE CAPPED GOLD

NANOSPERES

B NANOSPHERES

C NANOSTARS
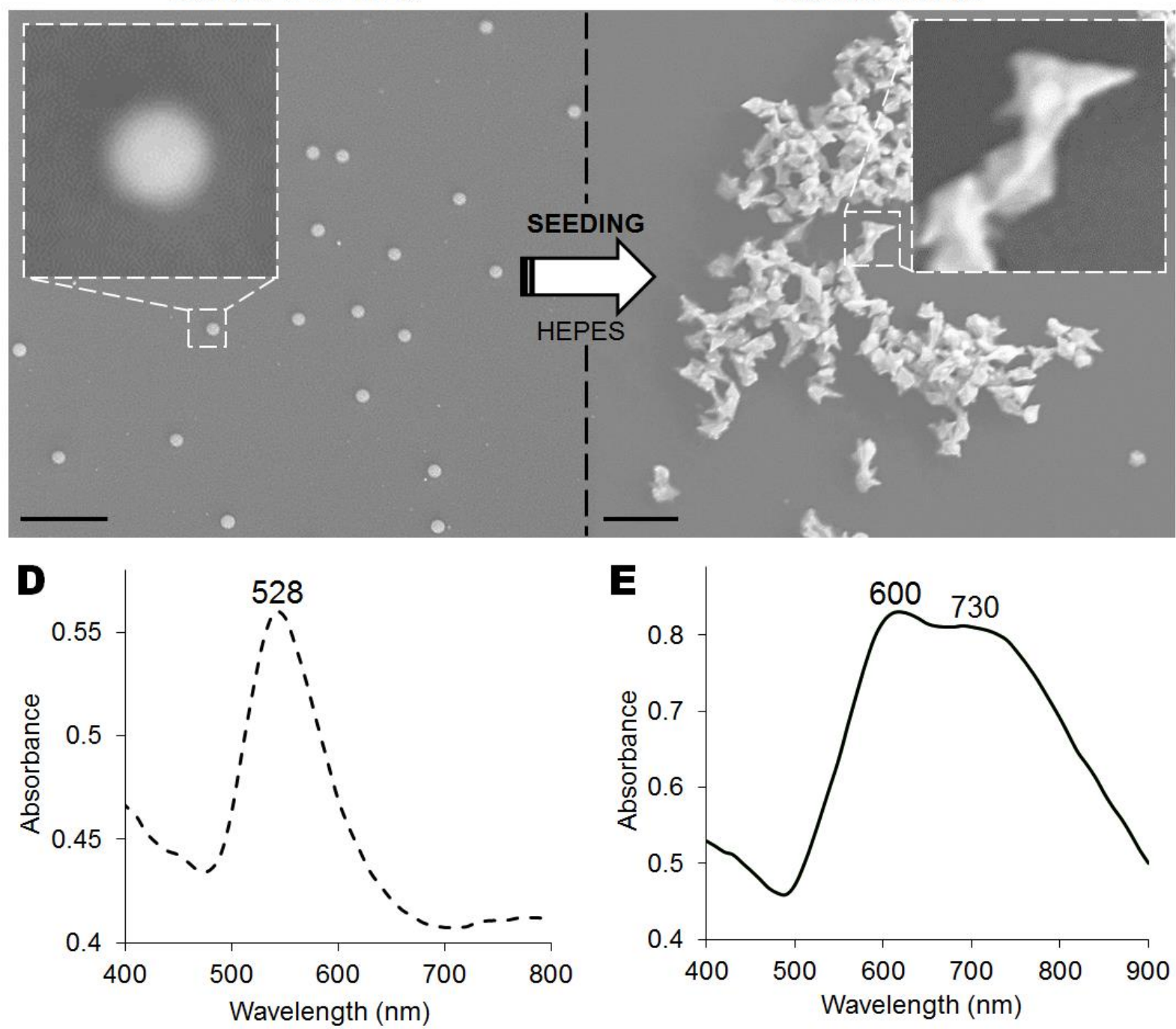

Figure 2. (A) Synthesis of gold nanostars via seed mediated route using HEPES buffer. Gold nanostars were prepared by reducing $\mathrm{Au}^{3+}$ using $10 \mathrm{~nm}$ citrate capped gold nanospheres as seeds in HEPES (3-[4-(2-hydroxyethyl)piperazine-1-yl]propane-1-sulphonic acid) buffer, where HEPES is serving as both reducing and shape-directing agent. Then, nanostars were functionalized with thiolated(-SH) PEG by ligand-exchange $\mathbf{0}$ between the thiold groups and the citrate groups on the nanoparticles; and Mitoxantrone (MTX) by EDC/NHS coupling 2. Scanning Electron microscopy (SEM) images of nanosphere (B) and nanostars (C) functionalized with MTX. UV-vis spectra of the same gold nanospheres (D) and 
nanostars $(\mathbf{E})$ in aqueous solutions. The extinction coefficient of nanostars is $6 \times 10^{8} \mathrm{M}^{-} 1 . \mathrm{cm}^{-1}$. Scale bars, $100 \mathrm{~nm}$.

The optical properties of the GNPs in suspension thus reflect the characteristics of the local fields associated with the surface plasmon resonance (SPR). The UV-Vis spectra of the gold nanospheres modified with MTX (MTX:Nanosphere molar ratio of approximately 45:1) show a typical SPR peak of around $528 \mathrm{~nm}$, consistent with a $10 \mathrm{~nm}$ GNP functionalized with MTX (Figure 2D). Besides aggregation, changes in surface charge, size, and morphology of particles are responsible for this change in SPR. In fact, nanospheres and nanostars were compared in terms of size and charge, revealing that nanospheres showed an average diameter of $15.5 \pm 3.1 \mathrm{~nm}$ as measured by dynamic light scattering (DLS) and negative charge (zeta potential $-20.2 \pm 2.5 \mathrm{mV}$ ), whereas the nanostars revealed a mean particle diameter of $35.6 \pm$ $3.6 \mathrm{~nm}$ and a negative charge (zeta potential $-24.3 \pm 1.8 \mathrm{mV}$ ).

More importantly, the stability of the gold nanostars solution with and without MTX functionalization was tested and remained stable up to one month in $4^{\circ} \mathrm{C}$. The hydrodynamic particle size and zeta potentials of the nanostars, as well as their SPR peaks were measured during 30 days (Table 1). As shown in Table 1, the size, charge and surface plasmon peaks remain stable for days with no significant changes over time. The stability of the MTXnanostars was also evaluated in the presence of increasing amounts of serum overtime with no significant effect on the MTX release from the nanostars for up to 30 days (Supporting Information Figure S3).

Table 1. Physico-chemical characteristics of Nanostars and Nanostars+MTX for 30 days.

\begin{tabular}{|c|c|c|c|c|c|}
\hline & Time & $\begin{array}{l}\text { Hydrodynamic } \\
\text { Diameter }(\mathbf{n m})\end{array}$ & $\begin{array}{c}\text { Zeta } \\
\text { Potential (mv) }\end{array}$ & $\lambda_{\mu \alpha \xi}(\mathbf{n m})$ & $\lambda_{\mu \alpha \xi}(\mathbf{n m})$ \\
\hline \multirow{7}{*}{ 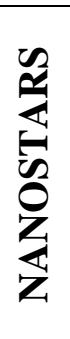 } & Day 1 & $35.5 \pm 2.3$ & $-19.1 \pm 2.1$ & $601 \pm 9$ & $729 \pm 11$ \\
\hline & Day 2 & $35.5 \pm 3.3$ & $-21.2 \pm 1.8$ & $602 \pm 11$ & $732 \pm 11$ \\
\hline & Day 3 & $35.1 \pm 3.4$ & $-22.3 \pm 2.1$ & $599 \pm 15$ & $733 \pm 13$ \\
\hline & Day 4 & $35.7 \pm 21$ & $-25.7 \pm 1.9$ & $601 \pm 17$ & $729 \pm 15$ \\
\hline & Day 5 & $35.4 \pm 2.8$ & $-22.2 \pm 1.7$ & $602 \pm 12$ & $728 \pm 13$ \\
\hline & Day 10 & $37.2 \pm 3.0$ & $-25.9 \pm 1.5$ & $601 \pm 18$ & $730 \pm 14$ \\
\hline & Day 15 & $35.8 \pm 3.7$ & $-25.1 \pm 1.5$ & $601 \pm 17$ & $733 \pm 21$ \\
\hline
\end{tabular}


BIOMATERIALS

\begin{tabular}{|c|c|c|c|c|c|}
\hline & $\begin{array}{l}\text { Day } 20 \\
\text { Day } 25 \\
\text { Day } 30\end{array}$ & $\begin{array}{l}35.5 \pm 3.2 \\
38.1 \pm 3.4 \\
37.7 \pm 3.2\end{array}$ & $\begin{array}{l}-25.2 \pm 1.7 \\
-25.5 \pm 2.2 \\
-22.5 \pm 2.1\end{array}$ & $\begin{array}{l}603 \pm 21 \\
598 \pm 17 \\
601 \pm 12\end{array}$ & $\begin{array}{l}729 \pm 17 \\
733 \pm 12 \\
730 \pm 15\end{array}$ \\
\hline \multirow{10}{*}{ 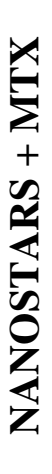 } & Day 1 & $38.2 \pm 1.3$ & $-23.2 \pm 1.5$ & $605 \pm 5$ & $732 \pm 5$ \\
\hline & Day 2 & $38.1 \pm 3.6$ & $-21.8 \pm 2.8$ & $606 \pm 10$ & $735 \pm 9$ \\
\hline & Day 3 & $38.5 \pm 4.4$ & $-23.2 \pm 2.4$ & $602 \pm 4$ & $731 \pm 8$ \\
\hline & Day 4 & $39.0 \pm 3.5$ & $-24.1 \pm 2.0$ & $602 \pm 7$ & $732 \pm 11$ \\
\hline & Day 5 & $38.1 \pm 1.8$ & $-23.5 \pm 2.1$ & $603 \pm 11$ & $730 \pm 14$ \\
\hline & Day 10 & $38.2 \pm 6.0$ & $-24.8 \pm 1.5$ & $601 \pm 8$ & $732 \pm 7$ \\
\hline & Day 15 & $38.7 \pm 4.9$ & $-24.3 \pm 2.8$ & $606 \pm 14$ & $733 \pm 15$ \\
\hline & Day 20 & $38.5 \pm 3.6$ & $-23.5 \pm 1.9$ & $604 \pm 8$ & $732 \pm 7$ \\
\hline & Day 25 & $39.2 \pm 2.5$ & $-23.9 \pm 2.4$ & $610 \pm 2$ & $736 \pm 13$ \\
\hline & Day 30 & $38.6 \pm 2.7$ & $-24.5 \pm 1.6$ & $605 \pm 10$ & $735 \pm 11$ \\
\hline
\end{tabular}

Regarding the optical properties of the GNPs, drug-loaded gold nanostars showed two SPR peaks: a transverse plasmonic resonance peak at $600 \mathrm{~nm}$, confirming the presence of a spherical core, and a longitudinal plasmonic resonance peak at $730 \mathrm{~nm}$, confirming the presence of the branch-shaped aggregation around the spherical core (Figure $\mathbf{2 E}$ ). The peak at $600 \mathrm{~nm}$ attributes from the spherical core and the peak at $730 \mathrm{~nm}$ attributes the peripheral vertices. The spherical core of nanostar results in a SPR similar to the nanospheres while the multiple vertices contribute SPR in the near infrared range. ${ }^{33,34}$

The Raman spectra of the MTX-loaded gold nanostars under different laser lines $(532,633$ and $785 \mathrm{~nm}$ ) were also analyzed (Figure 3A). Since the intensity of the returned Raman signal is inversely proportional to the fourth power of the excitation wavelength, shorter wavelengths like $532 \mathrm{~nm}$ can provide stronger Raman scattering; however, can also cause sample fluorescence, which may interfere with Raman signal. As local field hotspots, the sharp vertices nanostars under a near infrared excitation source can eliminate sample fluorescence from most organic molecules Therefore, the best signal-to-noise ratio (SNR) for the MTX gold nanostar was observed for the laser line at $785 \mathrm{~nm}$, as it largely avoids fluorescence but still returns a Raman signal sufficient to enable detection at a good SNR.

A stronger signal from MTX-nanostars inside the cells was observed when compared to the signal in the extracellular medium (Figure 3B). The signal is higher inside the cells once the 
gold nanostars are already abundant in the intracellular medium, changing the electric field distribution being the highest intensity located inside of the cell. This is a consequence of the cell containing gold nanostars acting as a focusing medium and the largest enhancement is located inside the cell. Even though different laser excitations can be in resonance inside or outside the cell, the intracellular medium containing the gold nanostars can change the spatial location of the maximum electric field providing selective amplification inside the cell. This represents a significant difference in terms of Raman spectroscopy due to the non-linear relation between Raman intensity and electric field. ${ }^{35}$ As shown in Figure 3B, Raman scattering was not observable under the measurement conditions employed in the presence of drug-loaded gold nanostars outside the cells at a source wavelength of $785 \mathrm{~nm}$. For the MTXnanostars inside the cells, three different groups of modes were observable, associated with aromatic $\mathrm{C}-\mathrm{C}$ stretching at 460 and $1300 \mathrm{~cm}^{-1}$ of the MTX. The vibrational modes observed in the SERS spectra (Figure 3B) are assigned to the corresponding vibrational modes, in accordance with the observations of SERS of the MTX on the surface of the gold nanostars. Consistent with literature, ${ }^{36}$ two Raman bands with strong scattering intensities are observed at 460 and $1300 \mathrm{~cm}^{-1}$. Ganbold et al. also show that MTX spherical GNPs present two surface plasmon bands corresponding to the transverse and longitudinal Raman scattering bands in the visible $(520 \mathrm{~nm})$ and the near-infrared regions, respectively. ${ }^{36}$ The authors also performed Raman measurements to identify whether MTX spherical GNPs are inside the cell, using characteristic vibrational bands of the drug molecules. However, MTX on spherical GNPs appeared to exhibit strong Raman intensities upon excitation at $633 \mathrm{~nm}$ through a resonanceenhancement process, whereas our MTX nanostars present the best SNR at $785 \mathrm{~nm}$. The aggregate spheres can cause a longitudinal band at $633 \mathrm{~nm}$, but the multiple vertices of the nanostars contribute SPR in the near infrared range.

These data prove that MTX-nanostars are optimal candidates for intracellular SERS detection and drug release using Raman/FTIR imaging and that behave as Raman scattering enhancers 
due to their tunable SPR and large number of hot spots on the surface associated sharp tips. The results are consistent with the requirement for enhanced electric fields at hotspots associated with the vertices of star shaped nanoparticles. ${ }^{13,37}$ Thus, gold nanostars are unique materials with an intense surface plasmon band that affords absorption in the near-infrared region. ${ }^{21-26}$
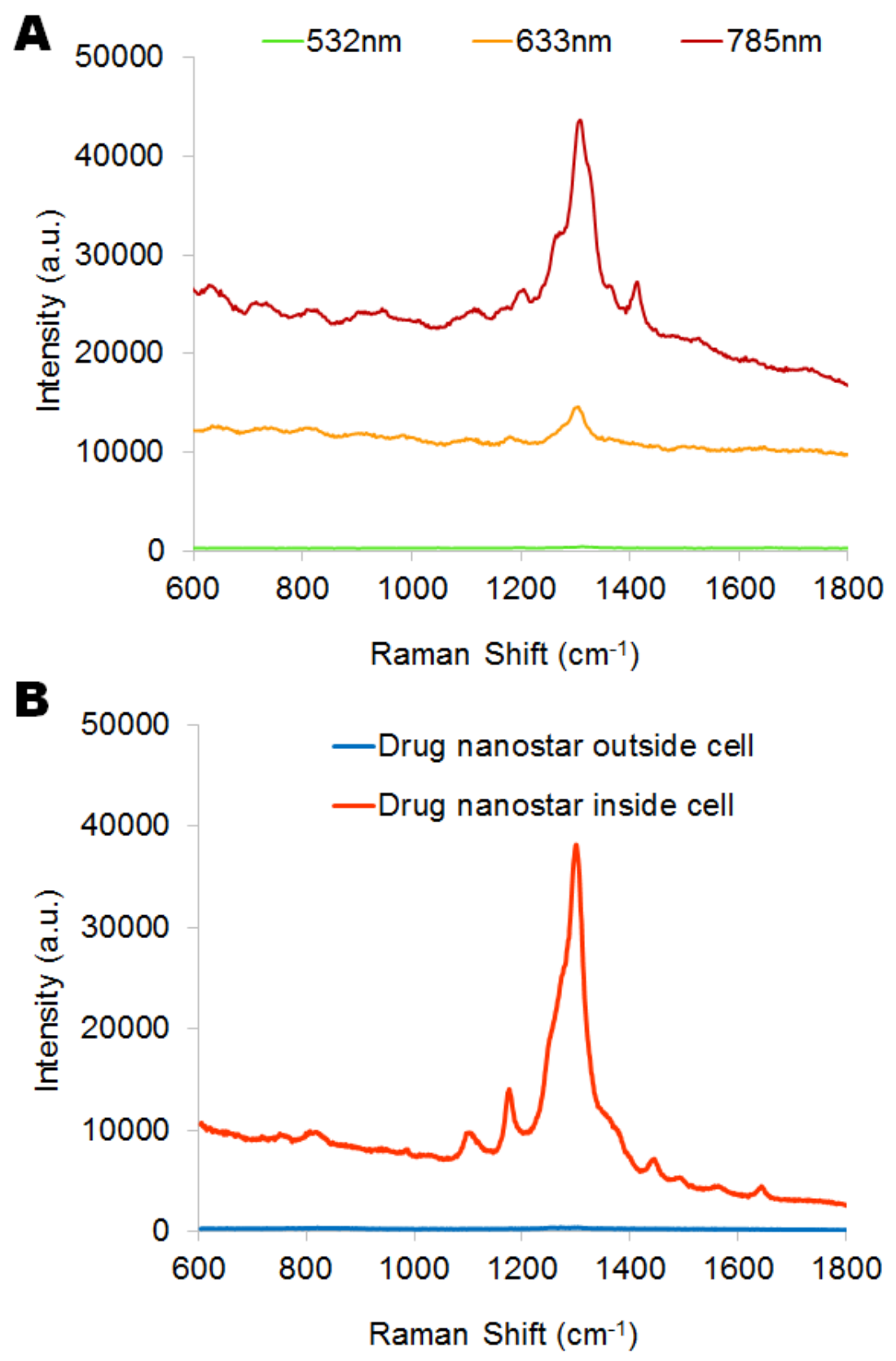

Figure 3. (A) SERS response of the gold nanostars under different laser lines (532, 633 and $785 \mathrm{~nm}$ ). (B) SERS signal inside and outside cell. The red line indicates the SERS signal of MTX-nanostars inside cell. The blue line shows the Raman spectrum of the MTX-nanostars outside the cells. 
To evaluate cellular uptake and biodistribution within cellular components and organelles, ultrastructural TEM images of human Caucasian lung epidermoid carcinoma cell (Calu-1 cells) further revealed that the MTX-nanostars were taken up by these cells in a larger extension than the bare nanostars, and were mainly localized in intracellular organelles (Figure 4A-B). After 24 hours of incubation, MTX-nanostars were found in the cytoplasm, clustered or monodispersed, demonstrating their capability to cross biological membranes and shuttle the MTX drug into the cytoplasm (Figure 4B).

Concerning biocompatibility, MTX-nanostars exhibited a reduced survival rate compared to nanostars alone. In a previous study, different shaped gold nanoparticles, such as spherical, triangular and hexagonal shaped have been investigated upon the cell survival rate of Calu-3 epithelial cells. The cytotoxicity was only observed after 72 hours following exposure of up to $1 \mathrm{mg} / \mathrm{mL}$. In the current study, no significant $(p>0.05)$ changes in cell viability were observed for either particle compared to the negative control at all concentrations tested (Figure 4C). In contrast, a significant $(p<0.05)$ loss in human Caucasian lung epidermoid carcinoma cell (Calu-1 cells) viability was found following exposure to MTX-nanostar (50\% viable) for 72 hours at $1 \mathrm{mg} / \mathrm{mL}$ (Figure 4C), when compared to negative control levels. Noticeably, Calu-1 cells remained viable for both types of particles and concentrations of up to $0.2 \mathrm{mg} / \mathrm{mL}$ after 72 hours (Figure 4D). Further to the findings at $1 \mathrm{mg} / \mathrm{mL}$, the loss in cell viability observed after MTX-nanostar exposure was found to be significantly different $(p<0.01)$ to the status of the Calu-1 cells after exposure to the nanostar (Figure 4C). A concentration of $200 \mu \mathrm{g} / \mathrm{mL}$ MTX-nanostars was used for further in vivo studies. These findings clearly show that nanostars, in contrast to nanostar-MTX, elicited only limited, if not negligible cytotoxicity, whilst nanostar-MTX, at least at doses exceeding $200 \mu \mathrm{g} / \mathrm{mL}$, significantly affected cell viability. 

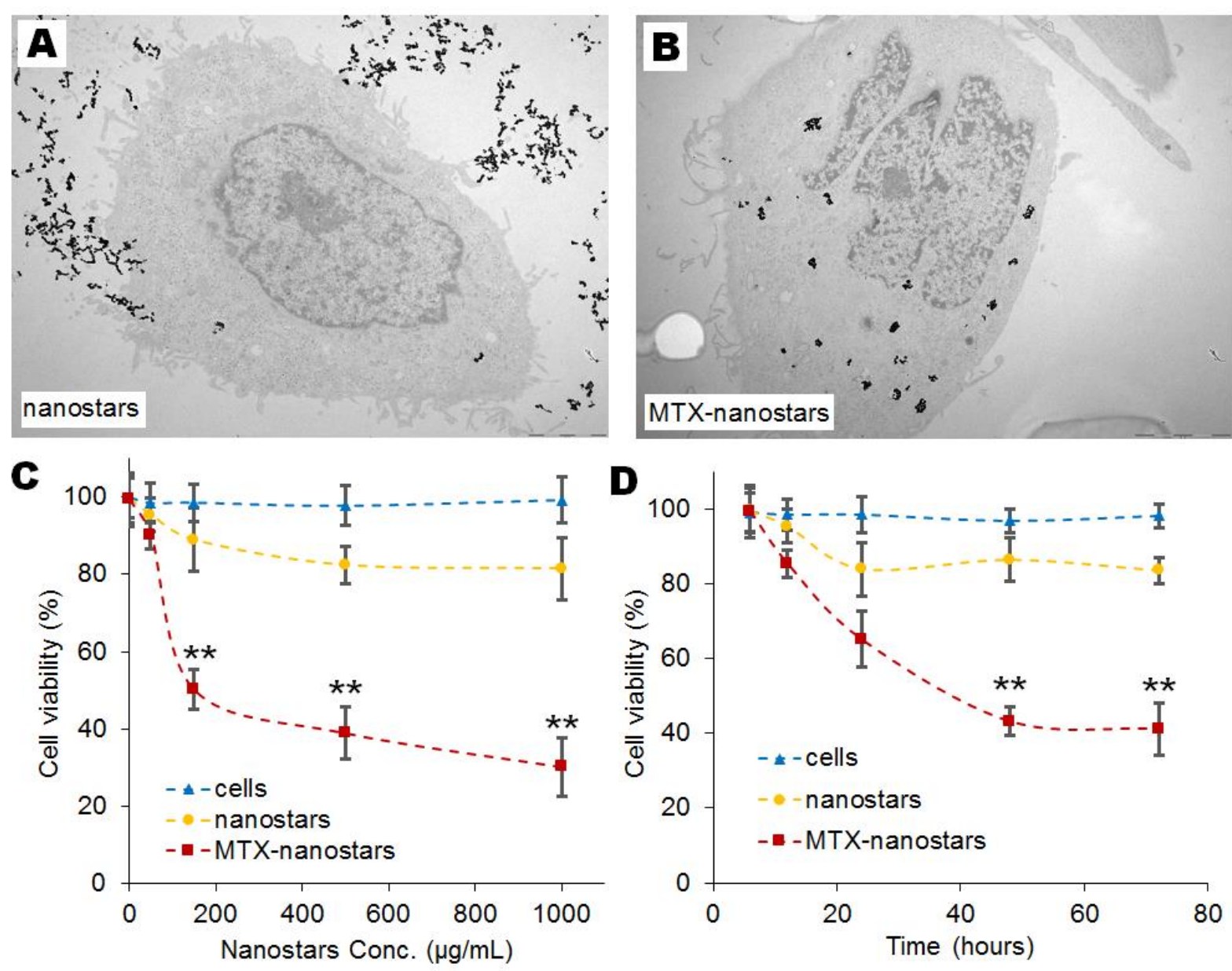

Figure 4. Nanostars cellular uptake and cytotoxicity in human Caucasian lung epidermoid carcinoma (Calu-1 cells). Ultrastructural TEM images of Calu-1 cells incubated with $200 \mu \mathrm{g} / \mathrm{mL}$ of nanostars (A) and MTX-nanostars (B) for 24 hours. Scale bars: A) $2 \mu \mathrm{m}$ and B) $5 \mu \mathrm{m}$. (C) Percentage of cell viability after 72 hours' exposure to increasing concentration of nanostars with and without MTX, when comparison with only cells. MTXnanostars cause cell viability to drop more than 50\%. (D) A similar trend was also noted for cell viability with increasing incubation times from 6 to 72 hours, using $0.2 \mathrm{mg} / \mathrm{ml}$ of nanostars with and without MTX. Data shown is the mean \pm standard error of the mean $(n=3)$.

In order to evaluate and characterize intracellular SERS detection and drug release, real-time Raman/FTIR and SERS maps were recorded for cells (human adenocarcinoma Calu-1 cells) with MTX-nanostars during 48 hours of incubation using the Perkin Elmer Spotlight 400 system for the FTIR and the LabRAM HR Evolution spectrometer for the SERS maps. Representative unprocessed FTIR and SERS maps, generated by the Spectrum image software, show spectrally averaged absorbance recorded from samples (Figure 5A), for 


\section{BIOMATERIALS}

several time points (1,8 and 48 hours). This allows to detect the modification of the wavelength of laser resulting in a fingerprint of information on the drug structure release. Nanoparticle regions are well defined in both the FTIR and SERS maps, due to their varying density and thickness. Spectra of the cells at 1, 8 and 48 hours of exposure to MTX-nanostars were extracted from the FTIR and SERS maps, evaluating the spectra of cellular structures characteristic of the cell line (see Figure 5B). Each spectrum was calculated as an average of 30-40 signals extracted from the FTIR and SERS maps characteristic of the defined cellular structure and was subjected to pre-processing using the RMieS-EMSC algorithm. ${ }^{38}$ The RMieS-EMSC algorithm is used to correct the infrared spectra and remove spectral distortions from Resonant Mie Scattering of biological cells. 

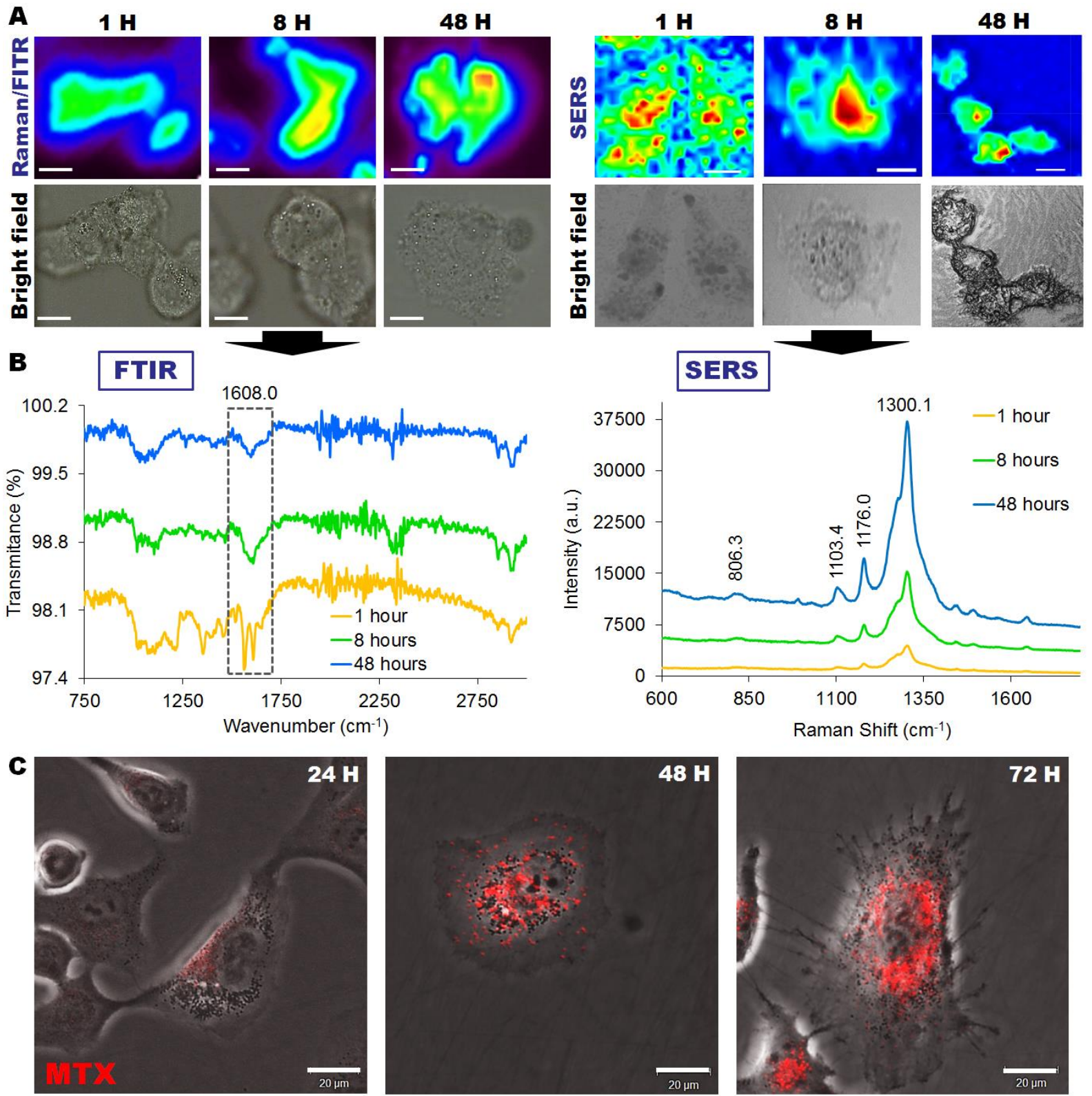

Figure 5. Real time monitory of MTX-Nanostars under FTIR and confocal microscopy imaging. (A) Real-time absorbance FTIR and SERS maps on Human Caucasian lung epidermoid carcinoma cells (Calu-1 cells) incubated with drug gold nanostars during 48 hours. Red color represents region of a nanoparticle, green and light blue indicate the background. Scale bars, $20 \mu \mathrm{m}$. (B) FTIR (on the left) and SERS (on the right) spectra of cells incubated with drug gold nanostars for 1,8 and 48 hours. (C) MTX-Nanostars under confocal microscopy from 24 to 72 hours of incubation. Scale bars, $20 \mu \mathrm{m}$.

The MTX-nanostars were observed under confocal microscopy from $24 \mathrm{~h}$ to $72 \mathrm{~h}$ (Figure 5C). The red color fluorescent dots (from MTX, emission maximum at $596 \mathrm{~nm}$, see Figure S2) indicated the MTX-nanostars spreading in cytoplasm. The amount of MTX released was estimated by both the increase in FTIR signal and the fluorescence intensity. Both FTIR and 


\section{BIOMATERIALS}

fluorescence intensity provide quantitative relationships between the spectral intensities of MTX concentrations in cells.

For in vivo SERS detection in healthy mice, live images of C57BL/6 mice were acquired from 1 to 5 minutes after IV injection of MTX-nanostars $(200 \mu \mathrm{g} / \mathrm{mL})$, showing a massive accumulation in the heart (Figure 6A). Ex vivo images of the heart at 5 min after IV injection are depicted in Figure 6B. This happens due to the presence of a highly organized vascular structure in the heart, favoring the accumulation of anticancer drugs within normal tissues over time, with a greater exposure to most normal tissues, such as heart. Indeed, Patel et al. has also reported that the distribution of anticancer drugs such as doxorubicin, mitoxantrone and topotecan from blood vessels is better in normal tissues, such as the heart and liver compared to the distribution in solid tumors. ${ }^{12}$ In fact, the biodistribution of MTX-nanostars was also confirmed by ex vivo organ inductively coupled plasma mass spectrometry (ICPMS) for 1 and 5 minutes after injection of the nanostars. The results show that 5 minutes after injection, MTX-nanostars are almost exclusively accumulated in the heart (consistent with the in vivo images in Figure 6A), with no significant accumulation in most of the other tissues and organs (Supporting Information Figure S4A). Kreyling et al. also show that nanoparticles distribute into heart at the first blood circulation and start to aggregate into lung after 4 hours. ${ }^{39}$ Our results also supports this idea that drugs are distributed in the blood, which is likely to provide greater access to well perfused organs such as the heart, liver and lung.

Average absorbance FTIR maps also showed evidence of hotspots due to the high intensity of MTX-nanostars on the heart tissue (Figure 6C). The color red represents region of the nanoparticle, green and light blue were the background. Hematoxylin and eosin (H\&E) staining on heart tissue was shown in bright field image (Figure 6D) in accordance with the FTIR maps (Figure 6C). The H\&E staining image can only show heart histological structure, but it is not sufficient for imaging of nanoparticles or small aggregates and not specific to 


\section{BIOMATERIALS}

anticancer agents. ${ }^{40}$ FTIR imaging does not require fixation, sectioning and staining as in

Figure 6C and the multiple vertices nanostars have the capability to enhance the MTX signal at the infrared range. FTIR maps can clearly provide the location and density of MTXnanostars in heart tissue at the same magnification view. Consequently, the experimental setting can reduce laboratory operations and maintenance costs.
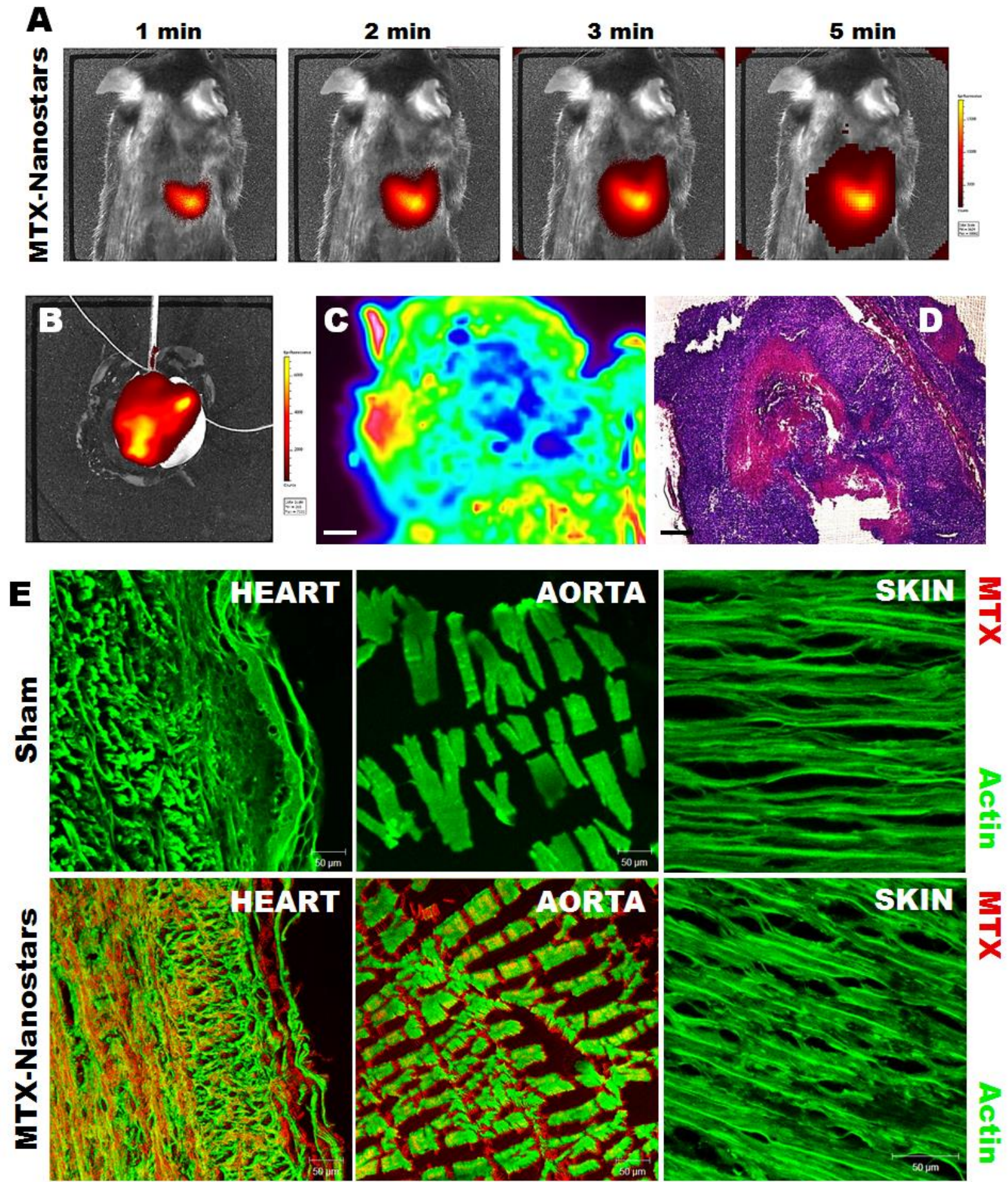

Figure 6. In vivo and ex vivo images of MTX-Nanostars in a healthy mice model. (A) In vivo live images of mice ( $\mathrm{n}=6$ animals) $1 \mathrm{~min}$ to $5 \mathrm{~min}$ after IV injection of MTX-Nanostars. 
(B) Ex vivo heart at 5 min after IV injection. (C) Average absorbance FTIR maps on heart tissue. Red color represents region of nanoparticle, green and light blue indicate background. (D) H\&E staining representing of heart tissue from the same sample provided in (C). Scale bars, $200 \mu \mathrm{m}$. (E) Representative confocal microscopy images of heart, aorta and skin tissue immunostaining from sham (no nanostars treatment) and MTX-nanostars treated groups, showing the biodistribution and accumulation of MTX-Nanostars in heart and aorta endothelium. Red color represents region of a MTX-Nanostars, green color shows actin staining.

In order to confirm the biodistribution of MTX-nanostars into the heart of healthy mice, immunocolocalization staining was performed (Figure 6E). The punctuated red color was represented the region of MTX-nanostars at laser line $514 \mathrm{~nm}$, the green color was shown the actin structure of endocardium and myocardium at laser line $488 \mathrm{~nm}$. No red color signal in the tissue of the control animal without MTX-nanostars was observed. The MTX-nanostars were located in among the green actin structure of the heart and aorta tissue (Figure 6E). The heart tissue and aorta tissue are permeable to MTX-nanostars due to high pressure of blood stream. Representative confocal microscopy images of heart, aorta and skin tissue confirm that the MTX-nanostars can cross the endothelial membrane in the aorta and heart. Moreover, no MTX-nanostars fluorescent signal was present on the skin located on the back of mice with the highest fluorescent intensity point under NIR imaging (no red color signal was located on the skin area, Figure 6E). The fluorescent signal on the back of the mice can be seen in Figure 6A. This data further provides evidence that MTX-nanostars biodistribution in heart and aorta is attributed to the fluorescent signal on the back side of mice (Figure 6A).

The biodistribution and accumulation in the heart is likely to be more efficient due to a highly organized vascular structure and large pressure gradients across the endothelial membrane. ${ }^{12}$ These results confirm this method as a great promise for application as a nonspecific antiinflammatory therapy for patients with cardiovascular diseases or chronic heart failure. In fact, MTX has also been used with success as a nonspecific anti-inflammatory therapy for patients with cardiovascular diseases or chronic heart failure. ${ }^{41}$ 
Concerning the in vivo SERS detection in a lung cancer mice model, we were able to image the characteristic SERS peaks and signal accumulated on the lung tumors of mice treated with MTX nanostars. The C57BL/6 mice bearing human Calu-1 xenograft lung tumor (3-mm diameter) received $0.2 \mathrm{~mL}(200 \mu \mathrm{g} / \mathrm{mL})$ of MTX nanostars. The MTX nanostars were administered in a single injection via tail vein. SERS spectra were taken 5 hours after injection. SERS measurements from the tumor tissue revealed the presence of MTX-nanostars (Figure 7). In order to test the development of the lung tumor in mice, bioluminescence imaging was carried out to serially assess lung tumor in each mouse treated with MTXnanostars, with luciferase activity as a measure for tumor burden (Figure 7A).

In order to track MTX-nanostars in tumor and lung tissues plasmonic-tunable Raman/FITR imaging spectroscopy was used (Figure 7B). The cancer tissue was under processed with standard cryosection steps using regular histology methodologies. The sections were cut and spread on $2 \mathrm{~mm} \mathrm{CaF}_{2}$ substrate, without any chemical treatment, in order to prevent any further chemical modification. Figure 7B shows the FTIR image of MTX-nanostars accumulated on lung tissue, revealing several tumoral clones in the lung. With this method we are able to image each individualized tumor in the lungs (arrows in Figure 7B). Imaging the lung tissue at different time points revealed that MTX-nanostars are uniformly distributed inside lung tissue and tumor at 5 hours after administration (Figure 7D), whereas ate 0.5 hours is still mainly around the tumor microenvironment (Figure 7C).

This experiment confirms the SERS signal in vivo and specificity of our MTX-nanostars. SERS mapping results confirms that MTX-nanostars are distributed more or less uniformly throughout the area of interest in the tumor tissue (Figure 7E). SERS mapping using characteristic peak (Figure 7F) can reveal the localization and distribution of the MTXnanostars throughout the anatomical location of the lung tumor tissue.

The biodistribution of MTX-nanostars was also confirmed by ex vivo organ inductively coupled plasma mass spectrometry (ICP-MS) for 0.5 and 5 hours after injection of the 
nanostars. The results show that 5 hours after injection, MTX-nanostars are mainly accumulated in the lung (tumor), with less accumulation in most of the other tissues and major organs, especially in the heart, liver and kidney (Supporting Information Figure S4B).
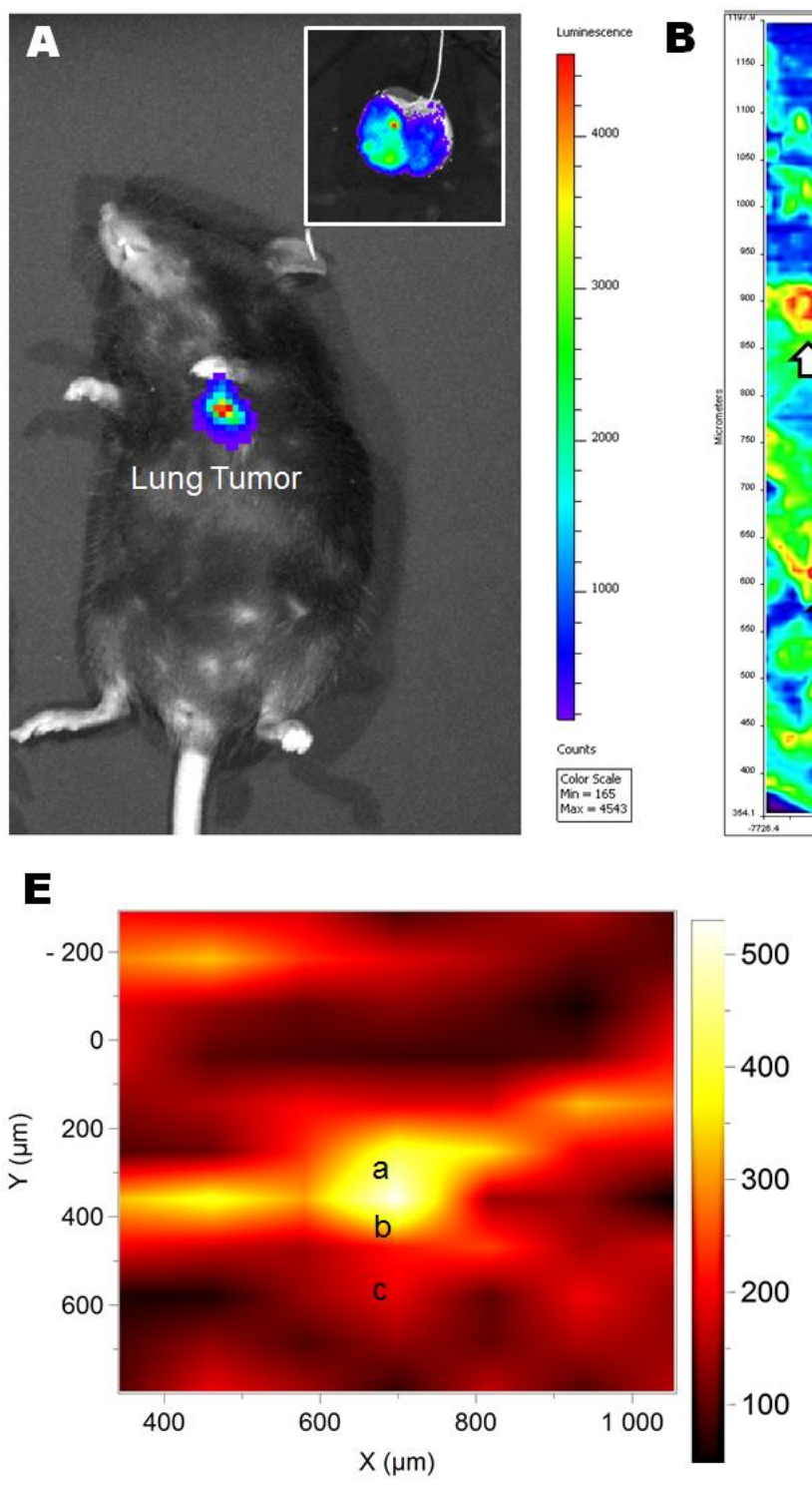
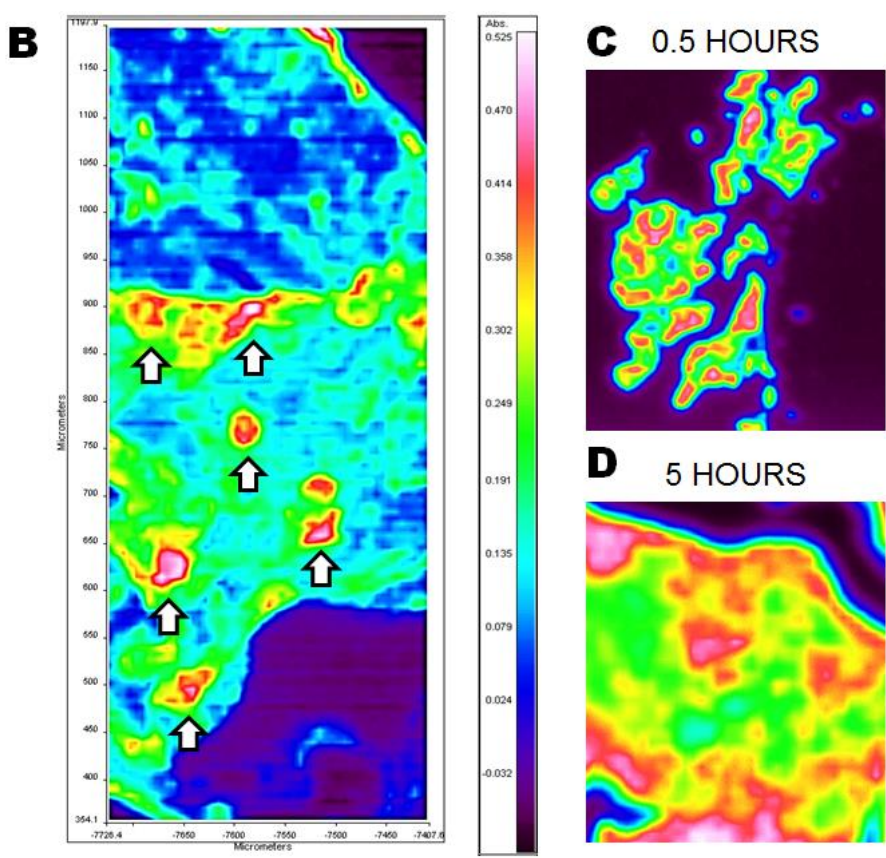

$\boldsymbol{F}$

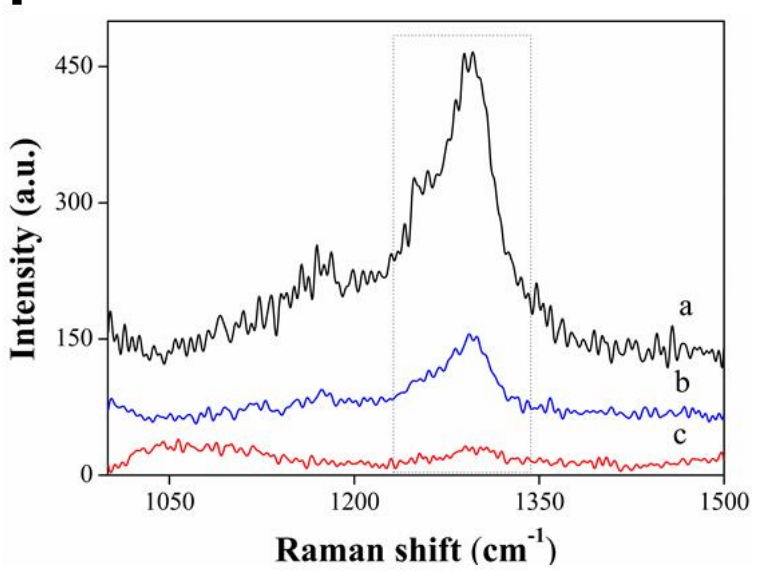

Figure 7. In vivo FTIR and SERS detection of MTX-nanostars in a lung cancer mice model ( $n=6$ animals). (A) Bioluminescent imaging of C57BL/6 mice bearing human Calu-1 xenograft tumor, to serially assed lung tumor development in each mouse, with luciferase activity as a measure for the tumor burden. (B) ATR-FTIR imaging of the lung tumor tissue for mice treated with MTX-nanostars. Arrows represent tumoral clones in the lung. ATRFTIR imaging of the lung tumor tissue for mice treated with MTX-nanostars after $0.5(\mathbf{C})$ and 5 hours (D) after administration. Scale bars, $200 \mu \mathrm{m}$. (E) SERS mapping image showing a laser beam focusing on the anatomical location of the lung tumor tissue $(400 \times 400 \mu \mathrm{m})$ based on intensity of the band at $1573 \mathrm{~cm}^{-1}$. (F) SERS spectra obtained from three different tumor tissue sites (a, b and $\mathbf{c}$ ). SERS spectra were measured at $785 \mathrm{~nm}$ excitation with 2-s signal integration. The spectra were background subtracted and shifted for better visualization. The 
colors and letters of the spectra correspond to the colors in $(\mathbf{E})$. The band at $\sim 1300 \mathrm{~cm}^{-1}$ is a characteristic band of the MTX-nanostars.

\section{CONCLUSION}

In summary, we propose a novel strategy based on plasmonic-tunable Raman/FTIR imaging spectroscopy, to directly track the delivery and release of an anticancer drug (Mitoxantrone MTX) by gold nanostars in single living cells and in two mice models: drug accumulation in the heart of healthy mice and in the tumor of lung cancer mice. Through the enhancement of the Raman signals from MTX molecules by the plasmonic field of the nanoparticles, we could simultaneously observe the anticancer drug scattering cellular imaging using the Raman scattering molecular vibration signals from molecules in living cells and in vivo, through a plasmonic-tunable Raman/FTIR imaging platform. This method proved to be extremely effective in detecting drug accumulation in both healthy and cancer tissue, being able to clearly individualize each tumoral clone in the lung, holding a great promise for application in image-guided cancer chemotherapy.

\section{METHODS}

Synthesis of gold nanostars via seed mediated route using HEPES buffer. 50-100 $\mu \mathrm{L}$ of $10 \mathrm{~nm}$ citrate capped gold colloid (Sigma Aldrich, Germany) was added to $12.5 \mathrm{~mL}$ of $30 \mathrm{mM}$ HEPES (3-[4-(2-hydroxyethyl)piperazin-1-yl]propane-1-sulfonic acid, Lonza) buffer (pH 7.0) cooled in ice bath with gentle stirring in a plastic container. $\mathrm{NH}_{4} \mathrm{OH}$ (Sigma Aldrich, Germany) was added to the reaction mixture to a final concentration of $0.4 \mathrm{mM}$. Stirring process was stopped before adding $2.5 \mathrm{~mL}$ of $1.0 \mathrm{mM}$ gold(III) chloride hydrate solution ( $\mathrm{HAuCl}_{4} \times \mathrm{H}_{2} \mathrm{O}$, Sigma Aldrich, Germany). The mixture was kept in ice bath protected from light until a color change was observed. The sample was stored immediately at $4^{\circ} \mathrm{C}$ protected from light.

Functionalization of HEPES-buffered gold nanostar through conjugation with PEG and Mitoxantrone. For the all functionalization steps and quantification assays of the nanostars with the PEG, can be found in Supplementary information. 
Characterization of functionalized gold nanostars. The stability of the gold nanostars solution was tested for up to one month in $4^{\circ} \mathrm{C}$ according to proceed. ${ }^{13}$ The diameter, Zeta Potential and Surface Plasmon Resonance peaks of the zeta potentials of the nanostars with and without MTX are depicted in Table 1.

Raman spectroscopy. Raman spectroscopy was performed with a HORIBA Jobin Yvon HR800 spectrometer with $532 \mathrm{~nm}, 632 \mathrm{~nm}$, and $785 \mathrm{~nm}$ diode laser as source according to proceed. ${ }^{13}$ Spectral data were collected using a $10 \times$ microscope objective over the range 400 $1800 \mathrm{~cm}^{-1}$ with a $10 \mathrm{sec}$ integration time. The detector used was a 16-bit dynamic range Peltier cooled CCD detector. Solutions were dropped onto $\mathrm{CaF}_{2}$ substrates and measured immediately. For comparison, spectra of the probe molecule MTX alone were recorded from $1 \mathrm{M}$ aqueous solutions.

Cell culture. The human adenocarcinoma (Calu-1) were cultured in complete culture medium of RPMI 1640 with L-Glutamine (Invitrogen, Ireland) supplemented with $10 \%$ (v/v) Fetal Bovine Serum (Sigma Aldrich, Ireland) and 1\% (v/v) $10000 \mathrm{U} / \mathrm{ml}$ Penicillin and $10000 \mathrm{U} / \mathrm{ml}$ Streptomycin (Invitrogen, Ireland). Cells were maintained on flasks at $5 \% \mathrm{CO}_{2}, 95 \%$ relative humidity and $37^{\circ} \mathrm{C}$. For subculture $0.25 \%$ trypsin and $0.03 \%$ EDTA solution were rained to remove the cell. Interval of cell passage were: every 6 to 8 days for all experimentation.

Cell viability assay. Nanostar and MTX-nanostars biocompatibility was evaluated in an epithelial cell line (Calu-1) over period 6 to 72 hours. In the WST-1 assay, cells were seeded on microplates at a concentration 4000 cell/well in $100 \mu$ culture medium containing GNPs. Different concentration of gold nanoparticle was loaded in the microplates at concentrations ranging from $0.0001-1 \mathrm{mg} / \mathrm{mL}$. The cells were incubated for $6,12,24,48$ and 72 hours at $37^{\circ} \mathrm{C}$ and $5 \% \mathrm{CO}_{2}$ after adding GNPS. A $10 \mu \mathrm{l} /$ well cell proliferation reagent WST-1 was mixture with cell medium and incubated for $4 \mathrm{~h}$ at $37^{\circ} \mathrm{C}$ and $5 \% \mathrm{CO}_{2}$. The samples were measured at the absorbance wavelength of $450 \mathrm{~nm}$. A triplicate analysis was performed from three independent experiments $(n=3)$.

In vitro application of Raman Spectroscopy. Calcium Fluoride $\left(\mathrm{CaF}_{2}\right)$ substrate was immersed in virkon for a few hours or overnight before plating the cells. The substrate was washed using sterilized water and placed in a small petri dish. Calu-1 cells were trypsinised and counted. A sufficient amount (approximately $500 \mu \mathrm{L}$ ) of cells suspension was added to cover the substrate. The dish was incubated in $\mathrm{CO}_{2}$ incubator for 3 hours. A fresh media (with $10 \%$ Fetal bovine serum, FBS) with nanostar was added to the $3 \mathrm{~cm}$ petro dish and the plates at final concentration of $0.2 \mathrm{mg} / \mathrm{mL}$ MTX-nanostars were left to be incubated for a period of $1,4,8,24,48$ and 72 hours. After the incubation period, the media in the dish is discarded and washed away using Phosphate buffered saline (PBS buffer). $3 \mathrm{~mL}$ of PBS buffer was added into the plate and the plate is ready to be measured using Raman spectroscopy.

Confocal microscopy imaging. Calu- 1 cells were seeded at a density of $1 \times 10^{4}$ cells $/ \mathrm{cm}^{2}$ and treated with $0.2 \mathrm{mg} / \mathrm{mL}$ MTX-nanostars in $3 \mathrm{~cm}$ petro dish for 24, 48 and 72 hours. Samples were then imaged by laser scanning microscopy (Carl Zeiss 510, at a 400× magnification). 
With each time points, four images per sample were captured to gain a representative understanding of drug location in Calu-1 epithelial cells following MTX-nanostars exposure.

FTIR Instrumentation. Six different cell samples and tissue slides samples were seed on the $\mathrm{CaF}_{2}$ crystal substrate to recording NIR spectra. NIR spectra were recorded with the Perkin Elmer Spotlight 400N Universal Attenuated Total Reflectance (UATR) accessory of the spectrometer. The spectral data were the result of 4 scans, with a spectral resolution of $8 \mathrm{~cm}^{-1}$.

Dynamic distribution of nanostar-MTX in mice. In order to better understand and predict drug effects and toxicities, drug distribution in time and location were recorded to complement pharmacokinetic data Briefly, MTX-nanostars $(0.2 \mathrm{~mL}, 10 \mathrm{mg} / \mathrm{kg})$ were injected into mice through the vena caudalis after chloroform inhalation. NIR imaging system equipped with a $765 \mathrm{~nm}$ laser as excitation source was employed to trace the distribution of the nanostar-MTX. The background image of the mice was taken prior to the injection. Due to a significant decrease in drug fluorescence after administration, a series of images were collected at 1, 2, 3 and 5 minutes post-injection respectively. All animal experiments were approved by the Institutional Animal Care and Use Committee of Shanghai Jiao Tong University (NO.SYXK2007-0025). The background fluorescence that was measured before injection was subtracted from the post injection fluorescence. In order to confirm the biodistribution of the nanostar in living mice, some major organs (heart, liver, spleen, lung, kidney, intestine of the subject mice were excised at 5 min post-injection and detected by NIR fluorescence imaging system.

Histology and immunolocalisation. After mice have been sacrificed and perfused with sterile PBS, heart, aorta and skin located at the back of mice with highest fluorescent intensity point under NIR imaging were snap-frozen in optimum cutting temperature compound (Tissue-Tek®) and cut on a cryostat microtome. Frozen sections were stained for hematoxylin and eosin using standard procedures. Stained tissue sections were then imaged by light microscopy. For immunolocalisation, the tissues sections were stained with a green phalloidin probe (Invitrogen) at a 1:40 dilution. Subsequently tissues were then imaged by ZEISS Confocal scanning microscopy at laser line $488 \mathrm{~nm}$ and $514 \mathrm{~nm}$.

In vivo SERS. MTX-nanostars $(0.2 \mathrm{~mL}, 10 \mathrm{mg} / \mathrm{kg})$ were injected into mice through the vena caudalis. The lung cancer tissue was directly carried out Raman imaging, and the flat area in the tissue was attractive candidate. Raman imaging was carried out in a LabRAM HR Evolution spectrometer (Horiba Scientific,) with a computer-controlled x,y stage in a $5 \mu \mathrm{m}$ step size at $633 \mathrm{~nm}$ excitation, using a 10x objective with a detection range from 1000-1600 $\mathrm{cm}^{-1}$. The excitation intensity and accumulation time was $5 \mathrm{~mW}$ and $2 \mathrm{~s}$, respectively. 
BIOMATERIALS

\section{ACKNOWLEDGEMENTS}

This work has been supported by Science Foundation Ireland 11/PI/1108 and a Marie Curie Intra European Fellowship, Grant Agreement n PIEF-GA-2012-332462 within the project “Gold nanoprisms (AuNPrs) for applications in Raman based lung cancer diagnosis". This work is also supported by the National Key Basic Research Program (973 Project) (No.2015CB931802), National Natural Scientific Fund (No. 81225010), 863 Project of China (2014AA020700), Shanghai Science and Technology Fund (No. 15DZ2252000).

\section{REFERENCES}

(1) Tong, S.; Fine, E. J.; Lin, Y.; Cradick, T. J.; Bao, G., Nanomedicine: Tiny Particles and Machines Give Huge Gains. Annals of biomedical engineering 2014, 42 (2), 243-259.

(2) Liang, X. J.; Chen, C.; Zhao, Y.; Wang, P. C., Circumventing Tumor Resistance to Chemotherapy by Nanotechnology. Methods in molecular biology 2010, 596, 467-488.

(3) Sperling, R. A.; Rivera, G. P.; Zhang, F.; Zanella, M.; Parak, W. J., Biological Applications of Gold Nanoparticles. Chem.Soc.Rev. 2008, 37 (9), 1896-1908.

(4) Giljohann, D. A.; Seferos, D. S.; Daniel, W. L.; Massich, M. D.; Patel, P. C.; Mirkin, C. A., Gold Nanoparticles for Biology and Medicine. Angew.Chem.Int.Ed Engl. 2010, 49 (19), 3280-3294.

(5) Bao, C. C.; Conde, J.; Polo, E.; del Pino, P.; Moros, M.; Baptista, P. V.; Grazu, V.; Cui, D. X.; de la Fuente, J. M., A Promising Road with Challenges: Where Are Gold Nanoparticles in Translational Research? Nanomedicine 2014, 9 (15), 2353-2370.

(6) Conde, J.; Dias, J. T.; Grazu, V.; Moros, M.; Baptista, P. V.; de la Fuente, J. M., Revisiting 30 Years of Biofunctionalization and Surface Chemistry of Inorganic Nanoparticles for Nanomedicine. Front Chem. 2014, 2, 48.

(7) Murphy, C. J.; Gole, A. M.; Stone, J. W.; Sisco, P. N.; Alkilany, A. M.; Goldsmith, E. C.; Baxter, S. C., Gold Nanoparticles in Biology: Beyond Toxicity to Cellular Imaging. Acc.Chem.Res. 2008, 41 (12), 1721-1730.

(8) Conde, J.; Tian, F.; Hernandez, Y.; Bao, C.; Cui, D.; Janssen, K. P.; Ibarra, M. R.; Baptista, P. V.; Stoeger, T.; de la Fuente, J. M., In Vivo Tumor Targeting Via Nanoparticle- 
Mediated Therapeutic Sirna Coupled to Inflammatory Response in Lung Cancer Mouse Models. Biomaterials 2013, 34 (31), 7744-7753.

(9) Conde, J.; Oliva, N.; Artzi, N., Implantable Hydrogel Embedded Dark-Gold Nanoswitch as a Theranostic Probe to Sense and Overcome Cancer Multidrug Resistance. Proc Natl Acad Sci U S A 2015, 112 (11), E1278-1287.

(10) Harmsen, S.; Huang, R.; Wall, M. A.; Karabeber, H.; Samii, J. M.; Spaliviero, M.; White, J. R.; Monette, S.; O'Connor, R.; Pitter, K. L.; Sastra, S. A.; Saborowski, M.; Holland, E. C.; Singer, S.; Olive, K. P.; Lowe, S. W.; Blasberg, R. G.; Kircher, M. F., SurfaceEnhanced Resonance Raman Scattering Nanostars for High-Precision Cancer Imaging. Sci Transl Med 2015, 7 (271), 271 ra277.

(11) Conde, J.; Ambrosone, A.; Sanz, V.; Hernandez, Y.; Marchesano, V.; Tian, F. R.; Child, H.; Berry, C. C.; Ibarra, M. R.; Baptista, P. V.; Tortiglione, C.; de la Fuente, J. M., Design of Multifunctional Gold Nanoparticles for in Vitro and in Vivo Gene Silencing. Acs Nano 2012, $6(9), 8316-8324$.

(12) Patel, K. J.; Tredan, O.; Tannock, I. F., Distribution of the Anticancer Drugs Doxorubicin, Mitoxantrone and Topotecan in Tumors and Normal Tissues. Cancer chemotherapy and pharmacology 2013, 72 (1), 127-138.

(13) Tian, F.; Bonnier, F.; Casey, A.; Shanahan, A. E.; Byrne, H. J., Surface Enhanced Raman Scattering with Gold Nanoparticles: Effect of Particle Shape. Anal Methods-Uk 2014, 6 (22), 9116-9123.

(14) Panchapakesan, B.; Book-Newell, B.; Sethu, P.; Rao, M.; Irudayaraj, J., Gold Nanoprobes for Theranostics. Nanomedicine (Lond) 2011, 6 (10), 1787-1811.

(15) Niidome, T.; Yamagata, M.; Okamoto, Y.; Akiyama, Y.; Takahashi, H.; Kawano, T.; Katayama, Y.; Niidome, Y., Peg-Modified Gold Nanorods with a Stealth Character for in Vivo Applications. Journal of controlled release : official journal of the Controlled Release Society 2006, 114 (3), 343-347.

(16) Hirsch, L. R.; Stafford, R. J.; Bankson, J. A.; Sershen, S. R.; Rivera, B.; Price, R. E.; Hazle, J. D.; Halas, N. J.; West, J. L., Nanoshell-Mediated near-Infrared Thermal Therapy of Tumors under Magnetic Resonance Guidance. Proc Natl Acad Sci U S A 2003, 100 (23), 13549-13554.

(17) Rodriguez-Oliveros, R.; Sanchez-Gil, J. A., Gold Nanostars as Thermoplasmonic Nanoparticles for Optical Heating. Opt Express 2012, 20 (1), 621-626. 
(18) Rodriguez-Lorenzo, L.; Krpetic, Z.; Barbosa, S.; Alvarez-Puebla, R. A.; Liz-Marzan, L. M.; Prior, I. A.; Brust, M., Intracellular Mapping with Sers-Encoded Gold Nanostars. Integrative biology : quantitative biosciences from nano to macro 2011, 3 (9), 922-926.

(19) Li, M.; Kang, J. W.; Dasari, R. R.; Barman, I., Shedding Light on the ExtinctionEnhancement Duality in Gold Nanostar-Enhanced Raman Spectroscopy. Angew Chem Int Ed Engl 2014, 53 (51), 14115-14119.

(20) Dam, D. H.; Culver, K. S.; Kandela, I.; Lee, R. C.; Chandra, K.; Lee, H.; Mantis, C.; Ugolkov, A.; Mazar, A. P.; Odom, T. W., Biodistribution and in Vivo Toxicity of AptamerLoaded Gold Nanostars. Nanomedicine 2015, 11 (3), 671-679.

(21) Vo-Dinh, T.; Fales, A. M.; Griffin, G. D.; Khoury, C. G.; Liu, Y.; Ngo, H.; Norton, S. J.; Register, J. K.; Wang, H. N.; Yuan, H., Plasmonic Nanoprobes: From Chemical Sensing to Medical Diagnostics and Therapy. Nanoscale 2013, 5 (21), 10127-10140.

(22) Liu, Y.; Yuan, H.; Kersey, F. R.; Register, J. K.; Parrott, M. C.; Vo-Dinh, T., Plasmonic Gold Nanostars for Multi-Modality Sensing and Diagnostics. Sensors (Basel) 2015, 15 (2), 3706-3720.

(23) Esenturk, E. N.; Walker, A. R. H., Surface-Enhanced Raman Scattering Spectroscopy Via Gold Nanostars. J Raman Spectrosc 2009, 40 (1), 86-91.

(24) Bi, S.; Jia, X.; Ye, J.; Dong, Y., Linear Light-Scattering of Gold Nanostars for Versatile Biosensing of Nucleic Acids and Proteins Using Exonuclease Iii as Biocatalyst to Signal Amplification. Biosens Bioelectron 2015, 71, 427-433.

(25) Liu, Y. et al. A Plasmonic Gold Nanostar Theranostic Probe for In Vivo Tumor Imaging and Photothermal Therapy. Theranostics. 2015, 5, 946-960.

(26) Yuan, H.; Khoury, C. G.; Hwang, H.; Wilson, C. M.; Grant, G. A.; Vo-Dinh, T., Gold Nanostars: Surfactant-Free Synthesis, 3d Modelling, and Two-Photon Photoluminescence Imaging. Nanotechnology 2012, 23 (7), 075102.

(27) Khlebtsov, N.; Dykman, L., Biodistribution and Toxicity of Engineered Gold Nanoparticles: A Review of in Vitro and in Vivo Studies. Chemical Society reviews 2011, 40 (3), 1647-1671.

(28) De Jong, W. H.; Borm, P. J., Drug Delivery and Nanoparticles:Applications and Hazards. Int J Nanomedicine 2008, 3 (2), 133-149.

(29) Xie, J. P.; Lee, J. Y.; Wang, D. I. C., Seedless, Surfactantless, High-Yield Synthesis of Branched Gold Nanocrystals in Hepes Buffer Solution. Chemistry of Materials 2007, 19 (11), 2823-2830. 
(30) Regev, R.; Yeheskely-Hayon, D.; Katzir, H.; Eytan G.D., Transport of anthracyclines and mitoxantrone across membranes by a flip-flop mechanism Biochem. Pharmacol. 2005, 70, 161-169.

(31) Homolya, L.; Orbán, T.I.; Csanády, L.; Sarkadi, B., Mitoxantrone is expelled by the ABCG2 multidrug transporter directly from the plasma membrane. Biochim Biophys Acta. 2011, 1808(1), 154-163.

(32) Shenkenberg, T.D.; Von Hoff, D.D., Mitoxantrone: a new anticancer drug with significant clinical activity. Ann Intern Med. 1986, 105(1), 67-81.

(33) Jin, R.; Cao, Y.; Mirkin, C. A.; Kelly, K. L.; Schatz, G. C.; Zheng, J. G., Photoinduced Conversion of Silver Nanospheres to Nanoprisms. Science 2001, 294 (5548), 1901-1903.

(34) Millstone, J. E.; Hurst, S. J.; Metraux, G. S.; Cutler, J. I.; Mirkin, C. A., Colloidal Gold and Silver Triangular Nanoprisms. Small 2009, 5 (6), 646-664.

(35) Mikoliunaite, L.; Rodriguez, R. D.; Sheremet, E.; Kolchuzhin, V.; Mehner, J.; Ramanavicius, A.; Zahn, D. R., The Substrate Matters in the Raman Spectroscopy Analysis of Cells. Sci Rep 2015, 5, 13150.

(36) Ganbold, E. O.; Yoon, J.; Kim, D.; Joo, S. W., Nonidentical Intracellular Drug Release Rates in Raman and Fluorescence Spectroscopic Determination. Physical chemistry chemical physics : PCCP 2015, 17 (5), 3019-3023.

(37) Chen, H.; Zhang, X.; Dai, S.; Ma, Y.; Cui, S.; Achilefu, S.; Gu, Y., Multifunctional Gold Nanostar Conjugates for Tumor Imaging and Combined Photothermal and Chemo-Therapy. Theranostics 2013, 3 (9), 633-649.

(38) Ostrowska, K.M., et al. Correlation of p16INK4A expression and HPV copy number with cellular FTIR spectroscopic signatures of cervical cancer cells. The Analyst, 2011, 136, 1365-1373.

(39) Kreyling, W. G.; Semmler-Behnke, M.; Seitz, J.; Scymczak, W.; Wenk, A.; Mayer, P.; Takenaka, S.; Oberdorster, G., Size Dependence of the Translocation of Inhaled Iridium and Carbon Nanoparticle Aggregates from the Lung of Rats to the Blood and Secondary Target Organs. Inhal Toxicol 2009, 21 Suppl 1, 55-60.

(40) Nedosekin, D. A.; Shashkov, E. V.; Galanzha, E. I.; Hennings, L.; Zharov, V. P., Photothermal Multispectral Image Cytometry for Quantitative Histology of Nanoparticles and Micrometastasis in Intact, Stained and Selectively Burned Tissues. Cytometry. Part A : the journal of the International Society for Analytical Cytology 2010, 77 (11), 1049-1058. 


\section{BIOMATERIALS}

(41) Gong, K.; Zhang, Z.; Sun, X.; Zhang, X.; Li, A.; Yan, J.; Luo, Q.; Gao, Y.; Feng, Y., The Nonspecific Anti-Inflammatory Therapy with Methotrexate for Patients with Chronic Heart Failure. Am Heart J 2006, 151 (1), 62-68. 